\title{
RARE CASE OF A HETEROZYGOUS MICRODELETION 9q21.11-q21.2: CLINICAL AND GENETIC CHARACTERISTICS
}

\author{
Ivanov $\mathrm{HY}^{1, *}$, Stoyanova $\mathrm{V}^{1,2}$, Ivanov $\mathrm{I}^{1,2}$, Linev $\mathrm{A}^{1,2}$, \\ Vazharova $\mathrm{R}^{3,4}$, Ivanov $\mathrm{S}^{4}$, Balabanski $\mathrm{L}^{4}$, Toncheva $\mathrm{D}^{4,5}$ \\ *Corresponding Author: Dr. Hristo Y. Ivanov, Department of Pediatrics and Medical Genetics, \\ Medical University Plovdiv, Vasil Aprilov 15-A Str., Plovdiv 4002, Bulgaria. Tel: +359-884-827-070. \\ Fax: +359-326-022-338. E-mail: doctorhristoivanov@yahoo.com
}

\begin{abstract}
Intellectual disability is affecting $3.0-4.0 \%$ of the general population. Copy number variants $(\mathrm{CNVs})$ are a significant cause leading to neurodevelopmental disorders such as intellectual disability, epilepsy, autism spectrum disorders and developmental delay. The use of single nucleotide polymorphism (SNP)-array and array comparative genomic hybridization (aCGH) as diagnostic tools has led to the recognition of new microdeletion/microduplication syndromes associated with neurodevelopmental disorders. It is also useful for further characterization of marker chromosomes. Here, we report a girl with mild intellectual disability and mild facial dysmorphisms. Cytogenetic analysis showed a marker chromosome in some percent of the cells and was followed by SNP-array karyotyping that detected, in addition, a $9655 \mathrm{Mb}$ de novo interstitial deletion at $9 \mathrm{q} 21.1-9 \mathrm{q} 21.2$.

Keywords: Deletion at 9q21.1; Interstitial deletion; Single nucleotide polymorphism (SNP)-array.

\footnotetext{
${ }^{1}$ Department of Pediatrics and Medical Genetics, Medical University Plovdiv, Plovdiv, Bulgaria

2 Department of Medical Genetics, University Hospital "St. George," Plovdiv, Bulgaria

${ }^{3}$ Department of Biology, Medical Genetics and Microbiology, Sofia University "St. Kliment Ohridski," Sofia, Bulgaria

${ }^{4}$ Department of Genomics, Hospital "Dr. Malinov," Sofia, Bulgaria

${ }^{5}$ Department of Medical Genetics, Medical University Sofia, Sofia, Bulgaria
}

\section{INTRODUCTION}

Copy number variants (CNVs) are a significant cause leading to neurodevelopmental disorders such as intellectual disability, epilepsy, autism spectrum disorders and developmental delay. Up to $3.0-4.0 \%$ of the general population is affected by intellectual disability, which makes it one of the most common neurological disorders [1]. Intellectual disability has been observed in patients carrying chromosomal aberrations or gene mutations.

Boudry-Labis et al. [2] proposed a novel microdeletion syndrome, involving a deletion at $9 \mathrm{q} 21.13$ and presenting with intellectual disability, speech delay, epilepsy, and characteristic facial features. They have reported 13 patients [2]. The range of the deletion spanned from 2.2 to $12.6 \mathrm{Mb}$ and included a different number of genes. Here, we report a girl with mild intellectual disability, speech delay and mild facial dysmorphism with 9q21.11-q21.2 microdeletion of about $9655 \mathrm{Mb}$ in size.

\section{CLINICAL REPORT}

The proband is a 10-year-old girl. She was referred to us for genetic consultation because of mild intellectual disability, speech delay and mild facial dysmorphism.

She was born full-term to non consanguineous healthy parents after an uneventful pregnancy with no evidence of asphyxia. At birth, weight, length, and head circumference were normal. There was no relevant family history. She had a history of global developmental delay in infancy. She walked at the age of 2 years. She said her first words at 18 
months of age and her first short sentence at the age of 2 years. At the age of 6 she was admitted to the hospital to establish a diagnosis regarding her developmental delay and short stature. She attends a mainstream school with teacher aide support.

On examination, her height was $135 \mathrm{~cm}$, weight 29 $\mathrm{kg}$ and head circumference $53.2 \mathrm{~cm}$. She had an elongated face with a prominent chin, broad forehead, arched bushy eyebrows and long eyelashes. She had hypertelorism and the palpebral fissures were down-slanting. Her nose had a broad prominent root. Her palate was narrow and highly arched. She had a thin upper lip, long philtrum, dystrophic teeth and excessive salivation. She had pes planus with large feet and short toes (Figure 1). After a walk of kilometer and a half she complained of cramp (pain) in her calves. A consultation with a child psychologist showed a mild intellectual disability (IQ 55, Wechsler Intelligence Scale for Children was used), cognitive deficits in attention with reduced volume, stability and concentration. Memory: difficulty in remembering and reproducing information. Thinking: clearly, figuratively. Cranial computed tomography (CT) scans and ultrasound of internal organs were performed and were normal.

Cytogenetic analysis was performed on cultured lymphocytes and skin fibroblasts by G-banding according to standard procedures. The SNP-array karyotyping was performed using microarray Illumina Human CytoSNP-12 (Illumina Inc., San Diego, CA, USA). The microchip contains a total of number of 301,232 SNP variants representative of the entire human genome. Genomic positions refer to the Human Genome February 2009 assembly (GRCh37/ hg19).

Cytogenetic analysis revealed a low-level mosaicism in blood and skin of the girl with a cell line carrying a small additional marker ring chromosome: $\operatorname{mos} 47, \mathrm{XX},+\operatorname{mar}[4] /$ 46,XX[96] (lymphocytes) and mos47,XX,+mar[16]/46,XX

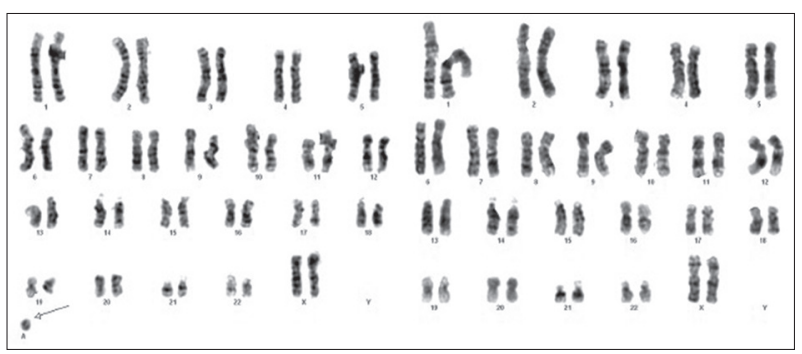

Figure 2. Karyotype of the proband.

[84] (skin fibroblasts). The mother had a normal karyotype. Chromosomal analyses of the father could not be carried out due to his lack of consent. (Figure 2).

For further characterization of the found marker chromosomes we applied SNP-array of genomic DNA from buccal swab, which showed a heterozygous partial deletion of the long arm of the chromosome 9 with size of about $9655 \mathrm{Mb}$. The deletion encompasses 43 human genes (Figure 3). To clarify the origin of the marker chromosome we offered fluorescent in situ hybridization (FISH) analysis, but the mother refused.

\section{DISCUSSION}

Here, we report a girl with a $9655 \mathrm{Mb}$ de novo deletion at 9q21.11-q21.2 presenting with mild intellectual disability, speech delay and characteristic facial features. Review of the literature showed that only 13 patients with a micro-deletion 9q21 have been reported [2,3]. The clinical symptoms observed in our patient are similar to those seen in the other patients with a deletion at 9q21.11-q21.2. In the cases described by Boudry-Labis et al. [2], they found mental retardation and speech delay in all patients, autistic behaviour, epilepsy and mild facial dysmorphism

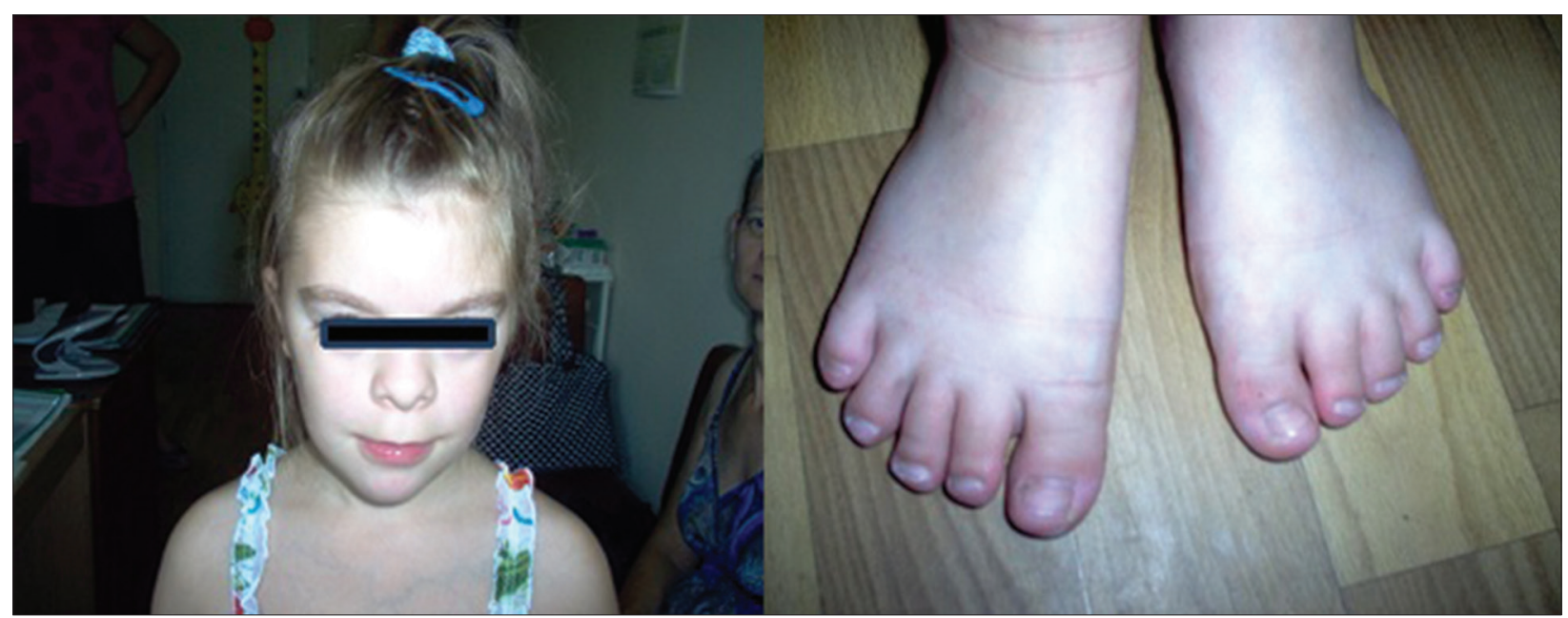

Figure 1. Phenotype of the proband. 


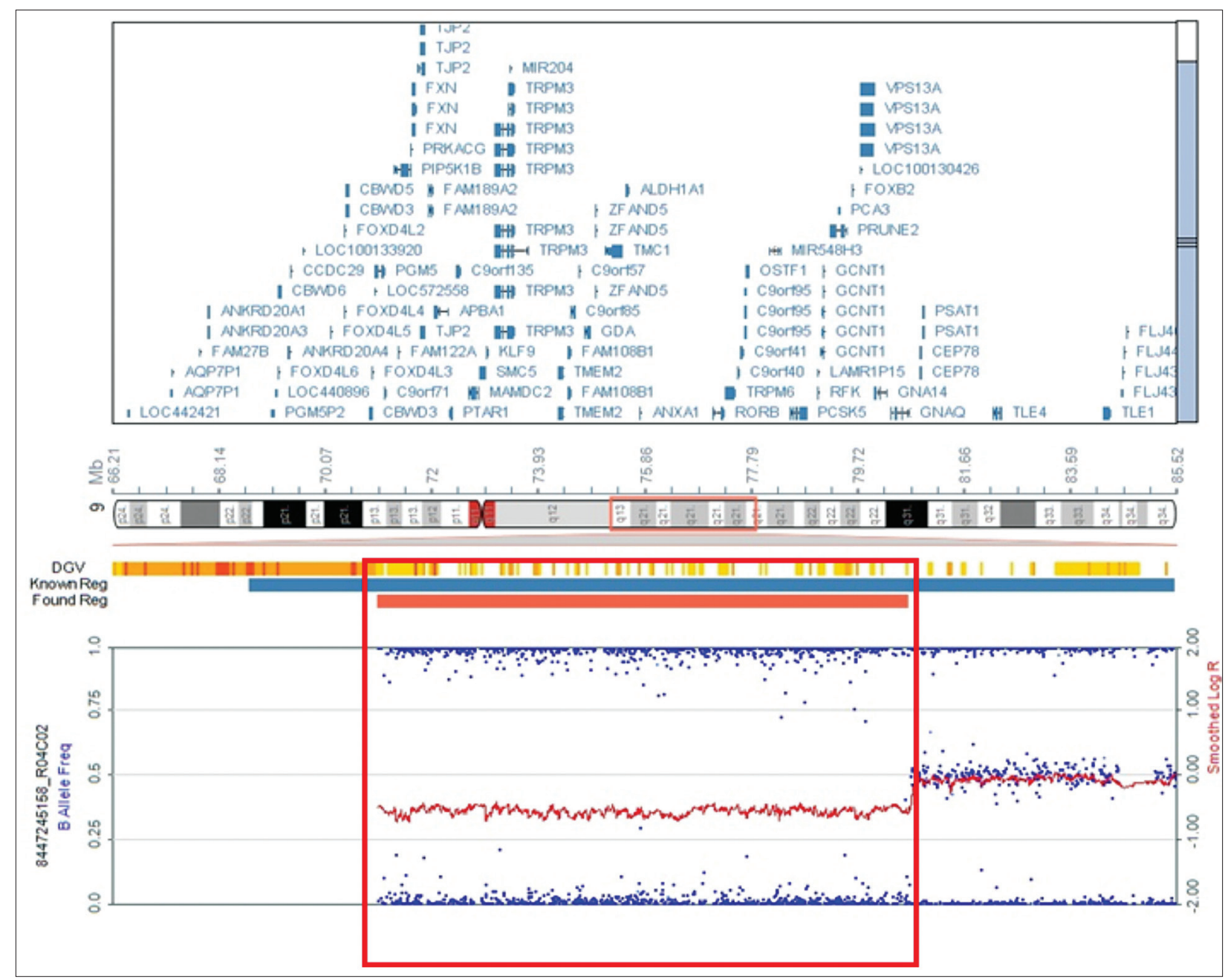

Figure 3. Graphical representation of chromosome 9 of the patient is shown. The deleted region of $9 \mathrm{q}$ is marked in red: $\operatorname{arr}$ [hg19] 9q21.11q21.2 (71034203-80689990) x1.

including hypertelorism, feature-less philtrum, thin upper lip, and in three cases, hypertrichosis. Our patient showed some of the phenotypic features of the cases described by Boudry-Labis et al. [2] but not all of them. She presented with mental retardation, speech delay, mild facial dysmorphism with hypertelorism, feature-less philtrum and thin upper lip but no epilepsy and autistic behavior.

Mosaicism in association with ring chromosomes is a well-known fact. Liehr et al. (4) summarized 144 cases, 78 of which $(54.0 \%)$ showed mosaic karyotypes. Thirtyone (out of 78) of the cases had no abnormal clinical findings. The non mosaic cases showed an even lower rate of abnormal clinical findings, in only 27 of 66 cases clinical abnormalities were described [4]. It is very difficult to interpret the influence of the market chromosome to the phenotype. Small marker ring chromosomes can be formed in association with a deletion of a part of the chromosome [4]. Due to the low-level mosaicism, molecular karyotyping could not identify the origin of the marker chromosome.
Thus, the contribution of the marker chromosome to the phenotype remains unknown.

Four genes (ROR $\beta$, PRUNE2, PCSK5 and TRPM6) located in the deleted segment are associated with a neurological phenotype, especially intellectual disability. From these, only TRPM6 is associated with a known human disorder (OMIM 602014). The other genes are not expressed in the brain and do not appear to be suitable candidates for neurodevelopmental disorders [2].

The $R O R \beta$ gene is expressed only in certain regions of the brain: the cerebral cortex, the thalamus, the hypothalamus, the pineal gland and the retina $[5,6] . R O R \beta$ null mice present with behavioral changes such as reduced anxiety behavior and several motor defects like lack of some neurological reflexes and abnormal gait. Some recent studies have established a strong genetic link between $R O R \beta$ and bipolar disorder [9] and the measure of verbal intelligence [10].

The PRUNE2 gene has involvement in neuronal apoptosis and is expressed in neurons in the brain, cerebel- 
lum, retina and spinal cord [11]. Recent studies suggested this gene as candidate gene to susceptibility to Alzheimer's disease and intellectual disability [11].

The PCSK5 gene is involved in transmitting of the nerve signals and is highly expressed in the nervous system, especially in the spinal cord and in the pineal gland. A recent study reported that zebra fish embryos lacking the coorthologue of the PCSK5 gene, PC5.1, have abnormal deposition of the neuromast within the lateral line system and have an abnormal touch response. This is consistent with the knowledge that the lateral line plays a role in the sensing the environment and in spatial awareness [12].

Mutations in the TRPM6 gene are associated with hypomagnesemia with secondary hypocalcemia (OMIM 602014). These patients presented with generalized convulsions or signs of increased neuromuscular excitability, such as muscle spasms or tetany, which can explain our patient's complaint of pain in the calves. In agreement with Boudry-Labis et al. [2], we could speculate that among the genes of the interstitial deletion at $9 \mathrm{q} 21.11-\mathrm{q} 21.2, R O R \beta$, PRUNE2, PCSK5 and TRPM6 could best explain the phenotype of our patient, characterized by mild intellectual disability and characteristic facial features.

In conclusion we can say that this case illustrates the need to implement high resolution methods in patients with intellectual disability and mild dysmorphism. Moreover, the genes associated with intellectual disability may warrant further investigation.

\section{ACKNOWLEDGMENTS}

Published with a grant under Project № BG05M2OP001-2.009-0025, "Doctoral training at MU-Plovdiv for Competence, Creativity, Originality, Realization and Academism in Science and Technology - 2 (DOCTORANT - 2)", funded under the Operational Programme "Science and Education for Smart Growth", co-funded by the Structural and Investment Funds of the EU

Declaration of Interest. The authors report no conflicts of interest. The authors alone are responsible for the content and writing of this article.

\section{REFERENCES}

1. Leonard H, Wen X. The epidemiology of mental retardation: Challenges and opportunities in the new millennium. Ment Retard Dev Disabil Res Rev. 2002; 8(3): 117-134.

2. Boudry-Labis E, Demeer B, Le Caignec C, Isidor B, Mathieu-Dramard M, Plessis G, et al. A novel micro- deletion syndrome at 9q21.13 characterised by mental retardation, speech delay, epilepsy and characteristic facial features. Eur J Med Genet. 2013; 56(3): 163-170.

3. Baglietto Mg, Caridi G, Gimelli G, Mancardi M, Prato G, Ronchetto P, et al. RORB gene and 9q21.13 mi-crodeletion: Report on a patient with epilepsy and mild intellectual disability. Eur J Med Genet. 2014; 57(1): 44-46.

4. Liehr T, Claussen U, Starke H. Small supernumerary marker chromosomes (sSMC) in humans. Cytogenet Genome Res. 2004; 107(1-2): 55-67.

5. Azadi S, Zhang Y, Caffe AR, Holmqvist B, Van Veen T Thyroid- $\beta 2$ and the retinoid RAR- $\alpha, \operatorname{RXR}-\gamma$ and ROR- $\beta 2$ receptor mRNAs; expression profiles in mouse retina, retinal explants and neocortex. Neuroreport. 2002; 13(6): 745-750.

6. Nakagawa Y, O'Leary DD. Dynamic patterned expression of orphan nuclear receptor genes ROR $\alpha$ and ROR $\beta$ in developing mouse forebrain. Dev Neurosci. 2003; 25(2-4): 234-244.

7. Andre E, Conquet F, Steinmayr M, Stratton SC, Porciatti V, Becker-Andre M. Disruption of retinoid-related orphan receptor $\beta$ changes circadian behavior, causes retinal degeneration and leads to vacillans phenotype in mice. EMBO J. 1998b;17(14):3867-3877.

8. Masana MI, Sumaya IC, Becker-Andre M, Dubocovich ML. Behavioral characterization and modulation of circadian rhythms by light and melatonin in $\mathrm{C} 3 \mathrm{H} /$ HeN mice homozygous for the ROR $\beta$ knockout. Am J Physiol Regul Integr Comp Physiol. 2007; 292(6): R2357-R2367.

9. Mcgrath CL, Glatt SJ, Sklar P, Le-Niculescu H, Kuczenski R, Doyle AE, et al. Evidence for genetic association of RORB with bipolar disorder. BMC Psychiatry. 2009; 9:70.

10. Ersland Km, Christoforou A, Stansberg C, Espeseth T, Mattheisen M, Mattingsdal M, et al. Gene-based analysis of regionally enriched cortical genes in GWAS data sets of cognitive traits and psychiatric disorders. PLoS One. 2012; 7(2): e31687.

11. Potkin SG, Guffanti G, Lakatos A, Turner JA, Kruggel F, Fallon JH, et al. Hippocampal atrophy as a quantitative trait in a genome-wide association study identifying novel susceptibility genes for Alzheimer's disease. PLoS One. 2009; 4(8): e6501.

12. Chitramuthu BP, Baranowski DC, Cadieux B, Rousselet E, Seidah NG, Bennett HP. Molecular cloning and embryonic expression of zebra fish PCSK5 coorthologues: Functional assessment during lateral line development. Dev Dyn. 2010; 239(11): 2933-2946. 